\title{
Ethical Issues in Genome Editing for Non-Human Organisms Using CRISPR/ Cas9 System
}

\section{Eduardo Rodriguez*}

Interdisciplinary Center for Studies on Bioethics, University of Chile, Chile

*Corresponding author: Eduardo Rodriguez, Interdisciplinary Center for Studies on Bioethics, University of Chile, Chile, Tel: 56-2-26031223; E-mail: erodriguezchi@gmail.com

Received date: March 21, 2017; Accepted date: April 03, 2017; Published date: April 04, 2017

Copyright: ( 2017 Rodriguez E. This is an open-access article distributed under the terms of the Creative Commons Attribution License, which permits unrestricted use, distribution, and reproduction in any medium, provided the original author and source are credited.

\begin{abstract}
Ethical issues in relation with genome editing for non-human organisms using CRISPR/Cas9 were reviewed. CRISPR/Cas9 system gives rise to significant ethical questions due to its accessibility; ability to make small, precise and specific edits of DNA in living cells; the ability of not leaving trace so that it is not possible to know whether the change has been introduced intentionally or through natural mutation; low cost; speed of use to produce results; and potential to achieve multiple simultaneous edits. The following issues were identified: risks of inducing mutations, the possibility of ecological disequilibrium for edited organisms release in the environment particularly in relation to gene drives, gaps in regulation, animal welfare, military or terrorist applications and the possibility of xenotransplantation between animals and humans. Bioethical issues are also discussed according to principles and about the relation with Nature. The following is recommended: public engagement and ethical reflection are needed to inform decision making; safety issues and environmental risk assessment must be enhanced to ensure efficacious regulation and preventions must be taken to oversee laboratories that use CRISPR technology.
\end{abstract}

Keywords: Genome editing; Ethics; Non-human organisms; CRISPR/Cas9

\section{Introduction}

For many years, molecular biologists have been looking for using cellular repair processes to intervene and modify DNA of organisms by guided genome editing; thus is, to change the organism genome by introducing a new function or correcting a mutation. Genome editing is defined as "the practice of making targeted interventions at the molecular level of DNA or RNA function, deliberately to alter the structural or functional characteristics of biological entities" [1]. The development of new approaches has made genome editing more precise, efficient, flexible and less expensive. Examples are the use of meganucleases; zinc finger nucleases (ZFNs) and transcription activator-like effector nucleases (TALENs). In the last years CRISPR/ Cas9 has been chosen as preferred method for genome editing due to its high degree of fidelity, relatively simple construction, rate of diffusion and low cost. The CRISPR/Cas9 system has many potential applications due to its ability to cut the DNA of any genome at any desired location by introducing the cas9 protein and appropriate guide DNA into a cell [2]. The sgRNA-Cas9 complex binds to its target and creates a double-strand break (DSB) that can be repaired by nonhomologous end joining (NHEJ) or by the homology-directed repair (HDR) pathway, modifying or permanently replacing the genomic target sequence. The system has the ability for genome editing and gene regulation in many types of organisms facilitating the function elucidation of target genes in biology and diseases. Genome editing has the potential to cure diseases by disrupting endogenous diseasecausing genes, correcting disease causing mutations or inserting new genes with protective functions $[3,4]$. It can be used to "knock out" genes or to change the function of genes by adding or replacing sections of DNA. Using CRISPR/Cas9 it is possible to make genome modifications in fertilized animal eggs or embryos altering the genetic makeup of every cell in an organism so that changes will be passed to following generations [5]. Another idea, which may encounter acceptation, is to recover extinct species [6]. Also, crops for human consumption may be improved with pest and disease resistant or better nutritional and other consumer qualities, with the advantage that by using CRISPR/Cas9 it is expected that the absence of foreign DNA in the final product and the introduction of genes derived from the same plant species should increase consumer acceptance [7]. CRISPR/Cas9 system gives rise to significant ethical questions due to its accessibility; ability to make small, precise and specific edits of DNA in living cells; the ability of not leaving trace so that it is not possible to know whether the change has been introduced intentionally or through natural mutation; low cost; speed of use to produce results; and potential to achieve multiple simultaneous edits.

Another line of research is to edit the genome of organisms to be used as models of human diseases, in which physiological and pathological processes could be studied in vivo [8,9]. Also it may facilitate transplanting animal organs into people by eliminating copies of retrovirus present in animal genomes that may harm human recipients [3]. Genome editing using CRISPR/Cas9 in combination with induced pluripotent stem cells may have the potential to enable the creation of human organs in animals, with the possibility of supplying organs not rejected by the immune system of human recipient and diminishing the risk of zoonosis [10]. These characteristics make this technique attractive to be used by any molecular biology lab, but the problem is that it can be used for any purpose unless be regulated. Scientific observations show concerned that the technology is developing too fast with little time for assessing ethical and safety issues associated with the use of this technology 


\section{Ethical Issues}

Ethical issues were ascertain in Medline articles and ethical guidelines of the Nutfield Council of Bioethics on the topic.

\section{Risk of inducing mutations}

As other genome editing, CRISPR/Cas9 system may induce offtarget mutations in the genome [11,12]. Mosaicism of the mutation(s) could be introduced when CRISPR/Cas9 system repeatedly target genes at different stages of embryonic development [13]. This may occur when CRISPR-Cas9 cleaves DNA sequences within the genome that are homologous to the target DNA sequences, causing an unintended mutation. Off-target mutations can be deleterious, causing cell death or transformation [14]. The technique has been improving with the goal to be used in a completely safe and efficient manner. Another issue is to improve the efficiency of homozygous knockout and homologous recombination, which is still low. The main challenge is to ensure the efficiency of genome editing while avoiding introducing changes in unintended parts of the genome. In this way, methods are being developed to reduce off-target effects and mosaic mutations and the efficiency is being improved [15]. Some scientists claim the use of a Cas9 protein engineered so efficient that no off target cutting is detectable across the whole genome in some organisms $[16,17]$, but every organism must be tested.

\section{Ecological disequilibrium}

The introduction of genetically modified organisms may provoke disequilibrium in ecosystems, since they may have advantages over related wild species. Furthermore, editing the Cas9 endonuclease gene with a gene encoding the desired guide RNA into an organism, adjacent to an altered gene, could create a "gene drive" that could spread a trait through an entire population of organisms, which may cause ecological disequilibrium. In 2015, a "gene drive" or so called "mutagenic chain reaction" was created in Drosophila, capable of driving a mutation in $97 \%$ of offspring in two generations, in which Cas9 endonuclease and the desire gene modification are inserted together into the target gene producing a mutagenic chain reaction [18]. Gene drive actively copies a mutation made by CRISPR on one chromosome to its partner chromosome ensuring that all offspring and subsequent generations will inherit the edited genome. Once introduced these genetic changes are self-propagated. If released outside a Laboratory, the effects will spread with every new generation. It has been envisioned that CRISPR technology may have the potential to eradicate disease vectors and invasive species, such as malaria, zika, chikungunya or dengue by targeting wild populations of diseasetransmitting organisms, but critics contend that they may appear unanticipated secondary effects [19]. For example, editing Aedes aegypti female mosquito may render it incapable of carrying dengue disease or inducing sterility in male mosquitoes preventing their reproduction, or limit the lifespan of their offspring. However, some researchers have demonstrated that using CRISPR/Cas9 is possible to create a gene drive that blocks malarial transmission without affecting mosquito populations by blocking Plasmodium genes [20]. Nevertheless, precautions must be taken since the method may have unintended environmental consequences, such as eliminating a food source for other species, or promote the proliferation of invasive pests or the sequence inserted may mutate and target unwanted parts of the genome. Gene drives have the capability to transmit mutations to next generations, but also the may transfer modified sequences or negative traits to related organisms. The ecosystem may be affected through community dynamics and ecological factors, depending on: role of the target species affected by gene drive in the community, species that fill a similar ecological niche in the community if the target species disappear, rapid changes in the community from one configuration to another affected by gene drive, effect over other species that have coevolved with the target species, development of mechanisms to neutralize the gene drive by target species, like evolving resistance [21]. The risk of broader ecosystem disruption is unknown and the duration of effects difficult to envision. Some researchers have warned about the risks and propose to implement ways of protecting against the accidental release of experimental organisms edited with gene drives [18]. The National Academy of Sciences [20] contents that there is insufficient evidence available at this time to support the release of gene-drive modified organisms into the environment, but it is possible highly controlled field trials. Experiments in the Laboratory must be done before releasing any edited organism to the environment under strict safety conditions and with appropriate safeguards.

The ability to design organisms with desired characteristics using CRISPR technology might encourage development in many laboratories without sufficient containment mechanisms, or result in the premature environmental release of those organisms and loss of control over their spread. Effects on the environment are more difficult to ascertain. Biosafety and biosecurity protocols need to be implemented. Before field testing or environmental release of organism modified by gene drive, it is necessary to know well the target organism, its relationship with the environment and possible consequences. The World Health Organization has outlined a phase testing pathway for testing genetically modified mosquitoes, which may be useful. The approach is precautionary, step by step, with careful study and evaluation, and checkpoints to determine whether to move to next phase and enhance their effectiveness [22].

\section{Issues of regulation}

Since genetically modified organisms has been produced for a long time, the CRISPR technology may not appear to create new ethical problems, but the affordability and efficiency of CRISPR technology create concerns about the generation and release of organisms edited by CRISPR/Cas9. There is need for effective and global regulation of genetically modified organisms, since current and international regulations provide inadequate guidance for CRISPR/Cas9 applications [23]. In the US, several agencies regulate genetically modified organisms, such as the FDA, the EPA, and the National Institutes of Health (NIH), but they lack sufficient control and monitoring capacity. The NIH has guidelines that require notification and containment procedures based on the pathogenicity, virulence, communicability, and environmental stability of organisms modified by CRISPR/Cas9, but lack regulatory authority and research not funded by the NIH is not subject to these guidelines. Today, many Biotechnology companies monitor the order of specific sequences from pathogenic organisms, but this is not done for all companies, not for private individuals [23]. Regulatory agencies should require that researchers demonstrate sufficient control mechanisms as a condition of using the CRISPR/Cas9 editing system. Internationally, the main regulatory instrument governing the development and use of genetically modified organisms is the United Nations Convention on Biological Diversity, implemented by the Cartagena and Nagoya Protocols, but some key players like the US, are not parties. Article 17 of Cartagena Protocol obligates parties to notify an International Biosafety Clearinghouse Committee and affected nations about any release of modified organism which may affect biological diversity or 
human health, but the document does not specify who will enforce the treaty, the need for prior testing, the limits on organism viability, methods to be used to assess effects and how to estimate damages or mitigate harms [23]. An International clearinghouse committee should be created where genetic sequence producers and sellers must register and where orders could be monitored.

There are concerns about patenting genome edited organisms. The critics of the patent law regime consider that the regime favors the private interests of biotechnological companies over public interest [24]. Litigations may occur also; for example, in the discovery of CRISPR/Cas9 system, there was a patent dispute between the two main claimants of intellectual property for the invention [25].

There is also need for regulating which limitations apply for the use of genome editing and for marketing genome editing products and for identifying organisms modified by genomic editing once in the market. Criteria needs to be establish for assessing the safety of CRISPR edited organisms for human consumption, and whether enhancement experiments will be accepted, for example: increasing the muscle mass of animals, enhance nutritional content, or create hornless cattle that are easier to handle [26]. Internationally, the Biological and Chemical Weapons Convention seeks to prevent research which seeks developing biological weapons, but there is no unified guidance for the modification of non-human organisms by gene editing for other purposes. Furthermore, this Convention has limitations to prevent the misuse of biological tools, since it has not been designed to address private companies or individuals, which as the technology moves forward may enter more as producers.

For long, crops have been genetically manipulated to make them less susceptible to disease and pests, more productive or more resistant to cold and dryness with the production of transgenics. Different from transgenics, CRISPR/Cas9 system no longer requires the insertion of foreign DNA into the plant genome using a virus, bacterial plasmid, or other vector system. For this reason, it has been challenged that CRISPR/Cas9 edited organisms do not classify as transgenic organism and therefore the legislation applied to transgenics may not apply for CRISPR/Cas9 edited organisms [23]. There is also the problem of not being able to detect DNA edition as different from a natural mutation in some cases, for which it is difficult the traceability of the edited organisms. However, there is a requirement for registering a commercial new plant variety for Plant Variety Protection to guarantee intellectual property rights for breeders. In the US, the Animal and Plant Health Inspection Service (APHIS), an arm of the US Department of Agriculture, has indicated that products resulting from CRISPR/Cas9 that only delete a gene, in most cases, would not be regulated because no new genetic material is integrated into the recipient genome. In recent years, APHIS has seen an increase in requests for non-regulation status by academic centers and biotechnology companies asking them to affirm that their products do not fall under current regulations, and so they avoid to be reviewed for safety and efficacy by federal agencies. On the other hand, the European Union regulation currently considers all genetically modified crops or animals as transgenic, regardless they result from the insertion of foreign DNA or direct genome editing, and therefore they are subjected to regulation and risk assessment. The regulatory system requires demonstration that there is no toxic or allergenic component added for being commercialized safely and that the new crop will not become a weed or threaten endangered or beneficial species under ecological perspective.
Gene drive experiments may fall under no particular regulation, except those that apply in general to recombinant nucleic acid research, but they may not fall under direct potential harm to humans or domesticated animals, which is more regulated. Scientists have called for strict biosafety measures and public review when it comes to introducing edited animals and insects into the environment [27]. Gene drives could be approved only if the safety and efficacy of performed edits have been rigorously tested and edited organisms should only be released to the environment after public consultation and appropriate consent of potentially affected populations with previous tests of ecological safety, including limits on organism viability and assessment of effects. Regulations should also require the development of methods to estimate damages and mitigate harms of edited organism when they prove harmful to other organisms, the environment, or humans. However, ecological risk assessments may not be sufficiently well developed up today to inform decisions about the use of gene drives.

\section{Animal welfare}

The potential suffering of sensitive, intelligent animals confined for life in Laboratory conditions to be tested with gene editing experiments and the killing of these animals requires ethical justification. There are risks to animal welfare for experimental animals due to technical difficulties in the use of gene editing. Due to off target mutations, there may be loss of function of a gene, adverse events, even fetal abnormalities. Public attitudes are moving towards rejecting research with non-human animals unless it is fully justified. The use of animals in research must be justified in terms of the research value for ameliorating animal disease, human disease or the understanding of fundamental biological processes. Scientists must consider whether experimental alternatives exist, whether the species used is appropriate, the number of animals used diminished, and methods to ameliorate or avoid suffering are used [28-30].

Another issue is the possibility of using genome editing technique to create personalized mutant animals as models for a personal health problem, since it may produce a different type of relation and responsibility towards the wellbeing of experimental animals [1]. These will not be studied for a general condition, but for a specific person, who will be especially concerned.

For animal wellbeing, the following considerations must be taken into account in experimental design for being sensible to their needs [31].

- The ability of animals to integrate groups with other member of their species through sight, smelling and contact.

- The design of the cage, avoiding dangerous materials or disease transmission.

- Availability and adequacy of elements that enrich environment.

- Intensity of animal manipulation and degree of damage of experimental procedures.

- Duration of confinement.

\section{Military or terrorist applications}

The design and building of new organisms or devices utilizing biological materials is the field of synthetic biology drawn from molecular biology, computer science, chemistry and engineering [32]. CRISPR/Cas9 is an enormously powerful tool for synthetic biology to 
generate microorganisms for a broad range of applications, from the production of pharmaceuticals, biofuels, or chemicals to the remediation of pollution or disease diagnostics and treatment, but the ability to design and edit whole genomes of bacteria and viruses with new properties raises concerns over military or terrorist applications. An important ethical issue is the possibility of using CRISPR/Cas9 editing system to synthesize and manipulate pathogens to make them dangerous for human health, even more potent. Experiments such as manipulation of the $\mathrm{H} 5 \mathrm{~N} 1$ strain of influenza virus to increase its transmissibility and virulence may harm society in relation to the risk it poses to human health [33]. Some publications are suspected of dual use, which means that under the apparent generation of new knowledge published, there is the idea of introducing lines of research with has the intention of producing harmful organisms for human health [34]. The ease and efficiency of CRISPR raises the concern that anyone with the appropriate equipment could engineer invasive species. The possibility of selling CRISPR/Cas9 kits affordable by a greater number of users beyond traditional biotechnological companies and research institutions challenge how this reality will be governed and regulated effectively.

\section{Xenotransplantation or humanized animals}

It is known the shortage of available human organs to replace damage organs for human health. For long, animal organs have been tried for transplantation, but there are risks of infection by animal microorganisms and rejection by the immune system, a risk which increases when species are discordant. Scientists are looking to enhance the possibility of transplanting organs from animals to humans using CRISPR technology by reducing the risk of zoonosis or transmission of microorganisms between animals and humans and reducing adverse immune response [35]. Multiple simultaneous editing followed by nuclear transfer cloning techniques or direct reprogramming of cells to gametes could potentially develop complex synthetic organisms or organic components for transplantation. Science, for example, published a report showing the inactivation of porcine endogenous retroviruses in pig kidney cells using CRSPR/cas9 with the goal of producing virus free transgenic pigs to grow organs for humans. But it is difficult to ascertain the complete removal of host pathogens, and there is concern that an unknown virus may potentially trigger new plagues through xenotransplantation.

Another idea to be explored is to create chimeric pigs unable to grow their own organs, introducing human stem cells so that these pigs grow human organs for xenotransplantation, but, a problem identified is that the formation of animal/human chimeras may carry human neural and germ cells [36].

In this topic there is also ethical concern over the pressure to use primates as organ donors, due to their similarity to humans.

\section{Bioethical issues}

Bioethics reflection favors the horizontal discourse between experts and lay people, which demand transparency in the information about benefits and risks of genome editing organisms.

The use of organisms for experimentation has as goal environmental cleaning, human consumption, saving human lives and ameliorating suffering through therapy, but this use imposes a moral responsibility to respect experimental organisms, not making them suffering unnecessarily, especially when working with sentient living beings. The current tendency is anthropocentric, considering human beings as the only moral subject with power to manipulate and use natural resources. The contrary tendency is called bioconservatism, frame in a reaction against human technological intervention in living beings which renders them non-natural, affecting their intrinsic value [1]. On the other hand, bioethical reflection moves to a moderate view, in which human being is able to transform nature, but nature has also power to modify human beings and any damage inflicted ends affecting also human beings [37]. The moral relevancy of non-human organisms relies on universal relations, since all organisms are connected, the earth itself could be considered alive which has achieved homeostasis equilibrium [38]. Under an eco-centric view, nature is a unitary being and any organism is defined through its relations with other organisms and the environment, giving them moral relevancy [37]. The interference with the balance of nature is particularly relevant when releasing modified organisms to the environment. Natural processes build stable ecosystems which may lose their balance under human intervention with potential catastrophic results.

There are some discussions regarding technological interventions to modify the genome of organisms since modifying their way of life may affect their intrinsic value. For example, an organism genetically modified may change its nutritional behavior or the way interacts with other organisms. One position holds that is wrong to intervene organisms artificially and other position holds that that the respect to non-human organisms imposes limits over the interventions of human beings [39]. Some scientists question whether intervening directly in the genome is different from other ways of manipulating nature, such as selective breeding of plants and animals, and generally is thought that the difference lies in the role in inheritance and the potential scale, seriousness and unpredictability of effects [1]. Others consider that there is a direct technological intervention modifying these beings, making them technological products subjected to patenting. In the discussion intervene beliefs, the sacredness of Nature, and there are power struggles for controlling the economic power of patenting. The global culture imposes a society move by profit in which the use of natural resources has no limit while there are benefits.

The precautionary principle set out in the Rio Declaration on Environment and Development (Principle 15) requires that reasonable measures should be taken to anticipate potential serious and undesirable consequences to the environment even without scientific proof. The precautionary approach should not only consider possible risks, but also possible benefits and possible harms of a range of alternative options and their effect over people, acknowledging uncertainties related to the complexity of the system and that the different sets of consequences may be valued differently by different people [1].

Furthermore, there is responsibility to future generations. Hans Jonas has reflected on the principle of responsibility for future generations, affecting not only human beings, but all living beings. We have responsibility so that future generations count with an environment biodiversity at least comparable to present [40]. Human beings have a great power over nature with genome editing technique and are responsible to take into account the risks. Ethically, there should be limits over human intervention. Also, living beings are not mere objects, subject to appropriation.

It is important to have an inclusive public sphere where to engage society on discussions about genome editing. Public engagement can help to frame and define the risks of gene-drive modified organisms and provide input into practical decision making and policy issues 
related to genome editing. The outcomes of public engagement may be as crucial as the scientific outcomes to decisions about whether to release a gene-drive modified organism into the environment. Engagement requires effort, attention, resources, and advanced planning.

\section{Conclusion}

While the use of CRISPR/Cas9 system may have many potential beneficial applications, it not should be overlooked that there are regulatory problems not fully solved. Of great concern is the possibility of using CRISPR/Cas9 with the intent of damaging others or the possibility of laboratories working with the technique lacking adequate containing mechanisms, so that experimental organisms may be released without testing the risks of damaging humans, other organisms or environment. Furthermore, ecological risk assessments are not sufficiently well developed to release organisms carrying gene drives.

Public engagement and ethical reflection are needed to inform decision making. Safety issues and environmental risk assessment must be enhanced to ensure efficacious regulation and preventions must be taken to oversee laboratories that use CRISPR technology.

\section{References}

1. Nutfield Council on Bioethics (2016) Genome editing: an ethical review.

2. Zhu LJ (2015) Overview of guide RNA design tools for CRISPR/Cas9 genome editing technology. Front Biol 10: 289-296.

3. Rath D, Amlinger L, Rath A, Lundgren M (2015) The CRISPR-Cas immune system: biology, mechanisms and applications. Biochimie 117: 119-128.

4. Carroll D (2014) Genome engineering with targetable nucleases. Annu Rev Biochem 83: 409-439.

5. Baltimore D, Berg P, Botchan M (2015) Biotechnology. A prudent path forward for genomic engineering and germline gene modification. Science 348: 36-38.

6. Shapiro B (2015) Mammoth 2.0: will genome engineering resurrect extinct species? Genome Biol 16: 228.

7. Kanchiswamy CN, Sargent DJ, Velasco R, Maffei ME, Malnoy M (2015) Looking forward to genetically edited fruit crops. Trends Biotechnol 33: 62-64.

8. Maddalo D, Manchado E, Concepcion CP (2014) In vivo engineering of oncogenic chromosomal rearrangements with the CRISPR/Cas9 system. Nature 16: 423-427.

9. Delerue F, Ittner LM (2015) Genome Editing in Mice Using CRISPR/ Cas9: Achievements and Prospects. Clon Transgen 4: 135.

10. Feng W, Dai Y, Mou L, Cooper DK, Shi D (2015) The potential of the combination of CRISPR/Cas9 and pluripotent stem cells to provide human organs from chimaeric pigs. Int J Mol Sci 16: 6545-6556.

11. Bortesi L, Zhu C, Zischewski J, Perez L, Bassié L, et al. (2016) Patterns of CRISPR/Cas9 activity in plants, animals and microbes. Plant Biotechnol J 14: 2203-2216.

12. Nekrasov V, Staskawicz B, Weigel D (2013) Targeted mutagenesis in the model plant Nicotiana benthamiana using Cas9 RNA-guided endonuclease. Nat Biotechnol 31: 691-693.

13. Luo X, Li M, Su B (2016) Application of the genome editing tool CRISPR/ Cas9 in non-human primates. Zoolog Res 37: 241-219.

14. Zhang F, Wen, Guo X (2014) CRISPR/Cas9 for genome editing: progress, implications and challenges. Hum Mol Genet 23: R40-46.

15. Koo T, Lee J, Kim JS (2015) Measuring and reducing off-target activities of programmable nucleases including CRISPR-Cas9. Mol Cells 38: 475-481.
16. Kleinstiver BP, Pattanayak V, Prew MS (2016) High-fidelity CRISPR-Cas9 nucleases with no detectable genome-wide off-target effects. Nature 529: 490-495.

17. Slaymaker IM, Gao L, Zetsche B (2016) Rationally engineered Cas9 nucleases with improved specificity. Science 351: 84-88.

18. Gantz VM, Bier E (2015) The mutagenic chain reaction: A method for converting heterozygous to homozygous mutations. Science 348: 442-444.

19. Oye KA, Esvelt K, Appleton E (2014) Biotechnology. Regulating gene drives. Science 345: 626-628.

20. Gantz VM, Jasinskiene N, Tatarenkova O (2015) Highly efficient Cas9mediated gene drive for population modification of the malaria vector mosquito Anopheles stephensi. Proc Natl Acad Sci USA 112: E6736E6743.

21. National Academies of Sciences, Engineering, and Medicine (2016) Gene drives on the horizon: advancing science, navigating uncertainty, and aligning research with public values. Washington, DC: The National Academies Press.

22. (2014) WHO (World Health Organization) The guidance framework for testing genetically modified mosquitoes. (World Health Organization, programme for research and training in tropical diseases).

23. Caplan AL, Parent B, Shen M (2015) No time to waste-the ethical challenges created by CRISPR: CRISPR/Cas, being an efficient, simple, and cheap technology to edit the genome of any organism, raises many ethical and regulatory issues beyond the use to manipulate human germ line cells. EMBO Rep 11: 1421-1426.

24. Piccioto S (2003) Private rights vs. public interests in the TRIPS agreement. Proceedings of the Annual Meeting (American Society of International Law) 97: 167-172.

25. Smith-Willis H, San Martín B (2015) Revolutionizing genome editing with CRISPR/Cas9: patent battles and human embryos. Cell Gene Ther Insights 1: 253-262.

26. Ledford H (2015) CRISPR, the disruptor. Nature 522: 20-24.

27. Esvelt KM, Smidler AL, Catteruccia F, Church GM (2014) Concerning RNA-guided gene drives for the alteration of wild populations. Elife 3: e03401.

28. Belmonte JCI, Callaway EM, Caddick SJ (2015) Brains, genes, and primates. Neuron 86: 617-631.

29. Blakemore C, Clark JM, Nevalainen T, Oberdorfer M, Sussman A (2012) Implementing the $3 \mathrm{Rs}$ in neuroscience research: a reasoned approach. Neuron 75: 948-950.

30. Russell WMS, Burch RL (1959) The Principles of Humane Experimental Technique. Methuen, London.

31. National Research Council (2011) Guide for the Care and Use of Laboratory Animals. National Academies Press, Washington DC.

32. Cameron DE, Bashor CJ, Collins JJ (2014) A brief history of synthetic biology Nat Rev Microbiol 12: 381-390.

33. Herfst S, Schrauwen EJA, Linster M (2012) Airborne Transmission of Influenza A/H5N1 Virus Between Ferrets. Science 1534-1541.

34. Lentzos F (2016) Dual use in biology and biomedicine, background paper commissioned by the Nuffield Council on Bioethics.

35. Fung RK, Kerridge IH (2016) Gene editing advance re-ignites debate on the merits and risks of animal to human transplantation. Intern Med J 46: 1017-1022.

36. Wu J, Okamura D, Li M, Suzuki K (2015) An alternative pluripotent state confers interspecies chimaeric competency. Nature 521:316-321.

37. Velayos C (1996) La dimensión moral del ambiente natural: ¿Necesitamos una nueva ética? Editorial Comares; Granada.

38. Lovelock JE (1985) Gaia, una nueva visión de la vida sobre la Tierra. Ediciones Orbis.

39. Nuffield Council on Bioethics (2015) Ideas about naturalness in public and political debates about science, technology and medicine.

40. Jonas H (1995) El Principio de Responsabilidad. Barcelona: Círculo de Lectores/Herder. 